\title{
Population Health Strategies to Support Hospital and Intensive Care Unit Resiliency During the COVID-19 Pandemic: The Italian Experience
}

\author{
Gabriele Romani, MD, ${ }^{1}$ Francesca Dal Mas, $\mathrm{PhD},{ }^{2}$ Maurizio Massaro, $\mathrm{PhD},{ }^{3}$ Lorenzo Cobianchi, MD, PhD ${ }^{4,5}$ \\ Mirko Modenese, $\mathrm{PhD},{ }^{6}$ Amelia Barcellini, MD, ${ }^{7}$ Walter Ricciardi, MD, MPH, MSc, ${ }^{8,9}$ \\ Paul Barach, MD, MPH, ${ }^{10-12, *}$ Rossella Lucà, PhD,,$^{13,14, *}$ and Maria Ferrara, MD ${ }^{15,16, *}$
}

\begin{abstract}
Italy was one of the countries most affected by the number of people infected and dead during the first COVID-19 wave. The authors describe the rapid rollout of a population health clinical and organizational response in preparedness and capabilities to support the first wave of the COVID-19 pandemic in the Italian province of Modena. The authors review the processes, the challenges faced, and describe how excess demand for hospital services was successfully mitigated and thus overwhelming the healthcare services avoided the collapse of the local health care system. An analysis of bed occupancy in the region predicted during the first weeks of the epidemic. The SEIR model estimated the number of infected people under different containment measures. Community resources were mobilized to reduce provincial hospitals' burden of care. A population health approach, based on a radical reorganization of the workflow and emergency patient management, was implemented. The bed saturation of the Modena Healthcare Agency was measured by an ad hoc, newly implemented intensive care unit (ICU) bed occupancy and COVID-19 centralized governance dashboard. ICU bed occupancy increased by $114 \%$, avoiding saturation of the Modena Healthcare Agency system. The EmiliaRomagna region achieved a higher rate of ICU bed availability at 2.15 ICU beds per 10,000 inhabitants as compared with community 1 ICU bed availability prior to the pandemic. Rapid and radical local reorganization of regional efforts helped inform the successful development and implementation of strategic choices within the hospital and the community to prevent the saturation of key facilities.
\end{abstract}

Keywords: COVID-19, intensive care units, community engagement, epidemic, public health strategies, health care management

${ }^{1}$ Hospital Health Direction, Public Health Agency, Azienda AUSL Modena, Modena, Italy.

${ }^{2}$ Department of Management, Lincoln International Business School, University of Lincoln, Lincoln, United Kingdom.

${ }^{3}$ Department of Management, Ca' Foscari University of Venice, Venice, Italy.

${ }^{4}$ Department of Clinical, Surgical, Diagnostic \& Pediatric Sciences, University of Pavia, Pavia, Italy.

${ }^{5}$ General Surgery Department, Fondazione IRCCS Policlinico San Matteo, Pavia, Italy.

${ }^{6}$ Humco s.r.l., Venice, Italy.

${ }^{7}$ Department of Radiation Oncology, National Center of Oncological Hadrontherapy (Fondazione CNAO), Pavia, Italy.

${ }^{8}$ Section of Hygiene, University Department of Health Sciences and Public Health, Catholic University of the Sacred Heart, Rome, Italy.

${ }^{9}$ Department of Woman and Child Health and Public Health, Public Health Area, Fondazione Policlinico Universitario A. Gemelli IRCCS, Rome, Italy.

${ }^{10}$ Wayne State University School of Medicine, Detroit, Michigan, USA.

${ }^{11}$ Jefferson College of Population Health, Philadelphia, Pennsylvania, USA.

${ }^{12}$ Interdisciplinary Research Institute for Health Law and Science, Sigmund Freud University Vienna, Austria.

${ }^{13}$ Institute of Biochemistry and Cell Biology - National Research Council (CNR), Rome, Italy.

${ }^{14}$ ISOPROG-Somatolink EPFP Research Network, Philadelphia, Pennsylvania, USA and Caltanissetta, Italy.

${ }^{15}$ Department of Psychiatry, Yale University, School of Medicine, New Haven, Connecticut, USA.

${ }^{16}$ Program for Specialized Treatment Early in Psychosis (STEP), Connecticut Mental Health Center, New Haven, Connecticut, USA.

*These authors contributed as co-last authors. 


\section{Background}

$\mathbf{T}$ HE NUMBER OF PEOPLE infected worldwide by the coronavirus COVID-19, has exceeded 74 million with more than 1.6 million dead globally (as of December 20, 2020). ${ }^{1,2}$ The first report of an Italian resident infected with COVID-19 was confirmed in the Northern Italian Lombardy region on February 21, 2020. ${ }^{3}$ On March 8, 2020, the Italian Government quickly mobilized and instituted case identification, containment, and allocated resources, to protect the health of the community including restricting citizen movement in many provinces in Northern Italy. A full lockdown of Italy was instituted on March 11, 2020.

The Italian health care system was quickly overwhelmed with more than 12,460 infected and 827 dead by March 11, 2020. Once the pandemic was recognized, the Italian Government enforced "anti-fragile" strategies aimed to stop the exponential spread of the infection, protect the most vulnerable citizens (ie, senior citizens, people with preexisting medical conditions), and support the health care system, already under enormous financial pressures, preventing its effective collapse. ${ }^{4-6}$

This article reports on the extensive public health planning and deployment efforts in the Modena, Emilia-Romagna province of Italy to successfully mitigate the predicted intensive care unit (ICU) and emergency department bed saturation at the beginning of the pandemic. The article reviews the challenges and barriers faced and proposes a planning and predictive health system strengthening model for health system leaders as they prepare to manage the next waves of the pandemic.

\section{Methods}

\section{Modena Province}

Italy has 60 million people. Each of the 20 Italian regional governments is independently responsible for overseeing their local Health Trusts and each is accountable for planning, delivering, and managing all health services. Modena Province has a catchment area of 701,642 people, and comprises 7 local Health Trusts. Hospitals in Modena provide 1800 beds and are organized in a hub-and-spoke model: 2 teaching hospitals, 5 general hospitals, and 5 private hospitals.

The Modena COVID task force was established on March 1, 2020. The Modena Province managed the crisis by creating new organizational structures and workflows to respond to COVID-19. The governance model required a rapid reorganization of services, physical spaces, and operational procedures to oversee and provide a seemless availability of staff, equipment, and available ICU beds. ${ }^{7,8}$

\section{Data sources}

The Modena Taskforce response included hundreds of action items, detailed in the Supplementary Data. International, national, regional, and local online sources of data were consulted in implementing a COVID plan (Supplementary Table S1). ${ }^{1,2,9-14}$ The new dashboard, Pagoda, was developed ad hoc to monitor all of the information concerning COVID-19 patients with scorecards and data dashboards (ie, bed occupancy, test positivity, etc.) The dashboard was used to retrieve the data for this analysis for the period February 24 to March 25, 2020.

\section{Data analysis}

Risk of exposure. The research team used the deterministic compartmental model SEIR, a classic infectious disease model that models the risks of exposure of people in 4 states: susceptible $(\mathrm{S})$, exposed $(\mathrm{E})$, infected $(\mathrm{I})$, and resistant $(\mathrm{R}){ }^{15-17}$ The basic assumption of the SEIR model is that all individuals (n) in the model will assume the 4 roles over time, meaning that $\mathrm{n}=\mathrm{S}+\mathrm{E}+\mathrm{I}+\mathrm{R}$. The SEIR model has some limitations for realworld situations, but it provides a good-fit model for simulating the course of different types of epidemics. The team estimated that the infected component (I) must be evaluated in different scenarios as a measure of risk: if there is no, partial, or a total quarantine.

The research team defined extreme scenarios using a model with progressively efficient containment measures. The outbreak was calculated estimating the effectiveness of the containment interventions (later on implemented by the Italian Government and still in use) from the data collected.

ICU bed occupancy. The ICU bed occupancy and availability in the Emilia Romagna and Lombardy regions were calculated by developing a statistical model forecasting hospital utilization against capacity as a crude number, and as a ratio between the most current total number of ICU beds available in a catchment of 10,000 inhabitants. A higher ratio meant greater ICU bed availability. Data were analyzed using Python software (Python Software Foundation, Wilmington, DE).

\section{Results}

\section{Models for estimating the number of infected people}

Figure 1 shows the different predictive scenarios regarding the spread of COVID-19 infection in the Modena Province by implementing containment measures from day 1 with varying degrees of effectiveness. The peak infections, with virus spread containment measures implemented at $60 \%$ effectiveness $(\rho=0.4)$, would have fallen on day 161 (August 8, 2020) with an estimated $2.9 \%$ of the population, equal to 161,000 infected people. However, a rise in the number of occupied ICU beds was already observed before the first containment measure was instituted on March 8 (Supplementary Figure S1).

\section{Resource allocation: logistics and operations}

The Modena Taskforce took an active role in directing the reallocation of resources to the hospitals and in the community, using a multi-level, multi-jurisdictional approach detailed in the following sections, and summarized in Figure 2.

Surge planning. The Modena Taskforce leaders saw a progressively increased utilization of hospital ICU beds across Modena (Supplementary Figure S1). The aim was to ensure ICU bed availability as the pandemic spread caused a massive increase in infected people. The surge was mitigated by reducing the number of patients requiring post-surgical beds, and by freeing up acute care practitioners (ie, physicians from surgery, emergency medicine, acute care). Ambulatory clinic spaces were converted to inpatient wards. Elective surgical and clinical nonurgent outpatient activities (eg, lab testing, imaging) were stopped, following lessons learned from the earlier influenza epidemic. ${ }^{18}$ All patients with a scheduled appointment were alerted by individual text message $(29,000$ 
SEIR model

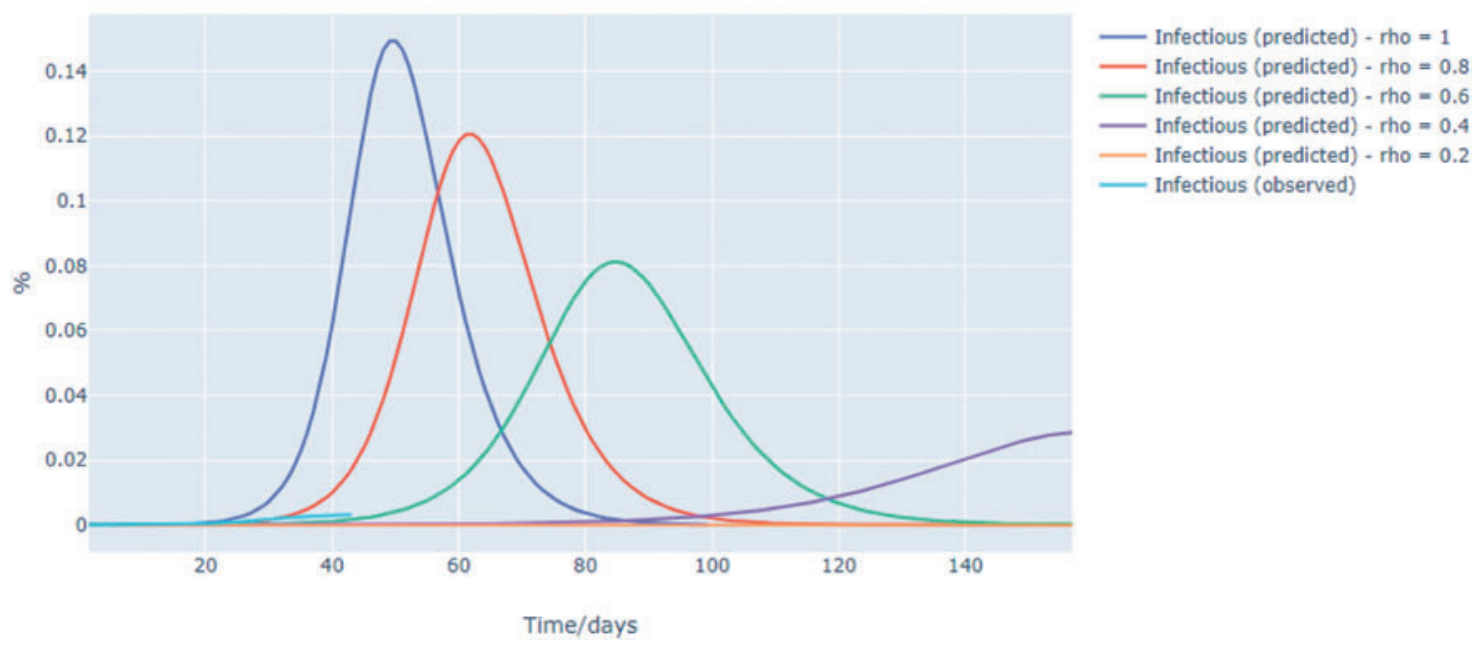

FIG. 1. Comparison of patient peak numbers according to the effectiveness of the containment interventions (rho). Variance on $\%$ of people infected (y-axis).

text messages were sent in the first 2 weeks, reaching $100 \%$ of those who had an appointment), and an aggressive public media and public health campaign was rolled out.

From February 20 to March 31, 2020, the number of ICU beds that became available for patient care in Modena province increased from 44 to 105 , while in the neighboring Emilia-Romagna region, they increased from 449 to 962 beds, making the ICU availability rate 2.15 beds per 10,000 inhabitants. The region was able to rely on existent available ICU beds and did not need the extra non-ICU beds that were

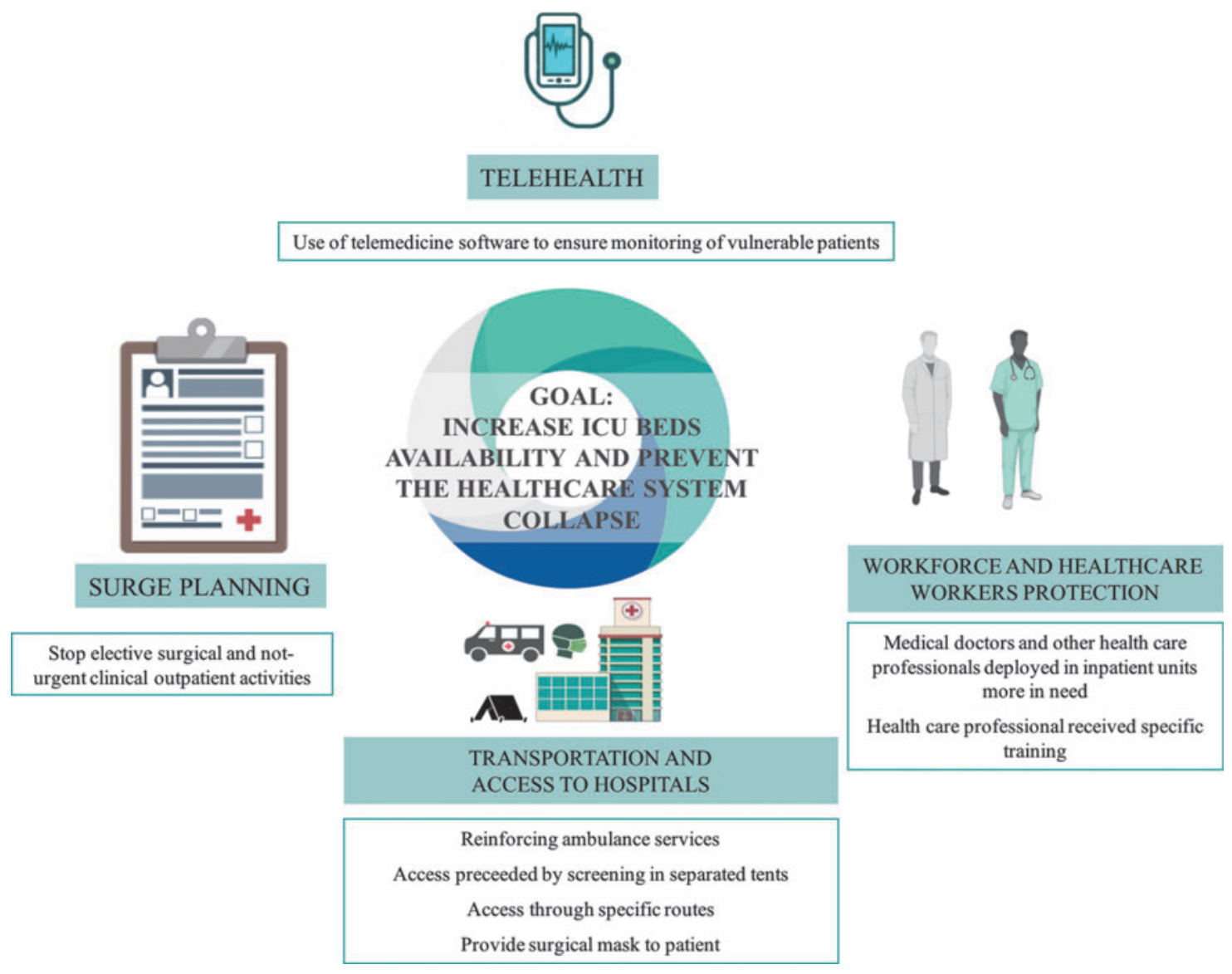

FIG. 2. Summary of Modena Taskforce logistics and operations. 
newly created. This is in contrast to the lack of available ICU beds in the nearby, equally hard hit, Lombardy region, which was quickly overwhelmed, leading to rationing of medical care for sick patients (Figs. 3a, 3b). Patients who needed invasive support with mechanical ventilation were admitted to ICU units or surgical operating rooms converted to ICU units. High-dependency care (noninvasive ventilation) was made available in converted infectious disease, emergency department, and internal medicine units.

Patient monitoring dashboard. A new data dashboard, Pagoda, was developed within 2 weeks from the start of the pandemic, and was implemented on March 16, 2020. The Pagoda dashboard was updated every 3 hours and was available to all clinical leaders in the province. Data were retrieved from a data warehouse run by the Modena Health Agency and included fully linkable demographic, hospital, emergency department, speciality, laboratory, and medication data. During the pandemic, Pagoda collected daily patient flow data from hospitals and ICUs, and collated the results of COVID-19 tests (Supplementary Figs. S2, S3). The dashboard improved considerably the ability of regional leaders to manage clinical resources, including staffing and ICU bed availability. The Pagoda software tool allowed for a rapid response to urgent requests for bed availability by regional hospitals: the facilitator or bed manager triaged the request and an ICU specialist was in charge of deciding the appropriate destination unit according to selected disease severity indexes.

Telehealth and remote patient monitoring. Previous work has described the potential to use telemedicine in disasters and public health emergencies. ${ }^{19}$ Strategies were quickly implemented to assure adequate remote care for patients with chronic non-COVID conditions. These included robust use of newly designed and adopted telemedicine software that enabled the use of video calls, and remote monitoring of physical health parameters, in order to assure the safe monitoring of vulnerable patients, such as those with mental and physical chronic illnesses. ${ }^{20,21}$ A specific COVID-19 telemedicine digital platform that allowed remote patient triage and monitoring service was implemented in the Modena long-term care residential facilities. Monitoring included a pulse oximeter connected via Bluetooth technology to a trackpad, which allowed the remote monitoring of blood oxygen levels and other vital signs such as heart rate, blood pressure, and body temperature (manually entered by the operator). Data retrieved were sent to the assigned primary care physician, and the digital platform also supported transmitting data to the closest emergency department.

Pregnancy care was assured using the Regional Birth Commission recommendations. ${ }^{22}$ Additionally, a multidisciplinary priority scale was developed and implemented to prioritize and support cancer patients. ${ }^{23}$

Transportation and access to health care facilities. Access to hospitals and emergency departments was only allowed for acute health emergencies and preceded by a detailed screening in adjacent newly erected tents. All patients were instructed on correct hand hygiene, and surgical masks were provided to all patients. Information on respiratory hygiene and cough etiquette was also provided (Supplementary Fig. S4).

Ambulance services were boosted to assure timely COVID-dedicated transfer of patients among units and to hospitals. Initially, 3 ambulances were dedicated to confirmed or suspected COVID-19 patients only; other ambulances were kept available for emergencies. However, later in the course of the epidemic, all regional ambulances were devoted to managing the increased COVID-19 patient care needs. Local industries donated more ambulances equipped for biocontainment transportation. A total of 3200 COVID19 ill patients were transported by ambulances from February 20 to April 21, 2020, a 6-fold increase in patient volume compared to the same period in 2019.
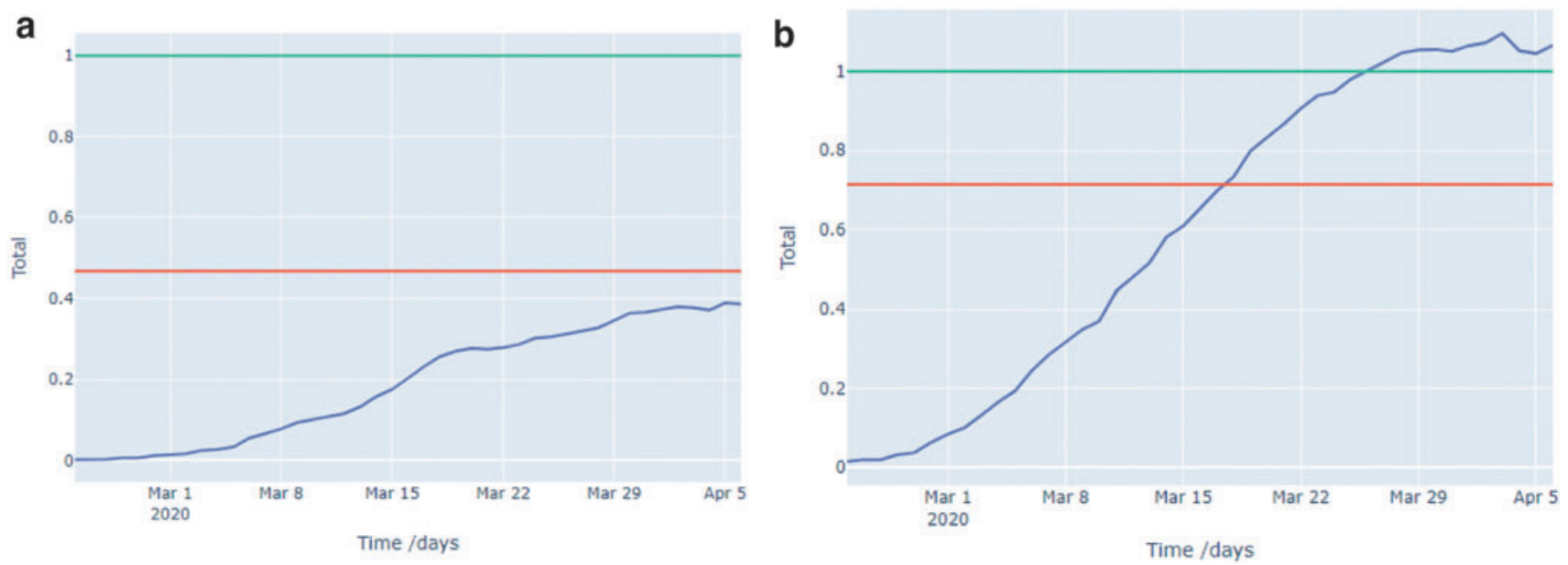

FIG. 3. Percentage of ICU bed occupancy, by time. In blue, the number of beds utilized. In red, current ICU beds available. In green, present and newly opened ICU beds. Figure 3a (left panel) Region Emilia-Romagna. Percentage of ICU beds occupancy (y-axis) by time. Total beds available: 962 (2.15/10K inhabitants). Figure 3b (right panel) Region Lombardy. Percentage of ICU beds occupancy (y-axis) by time. Total beds available: 1260 (1.25/10k inhabitants). ICU, intensive care unit. 
Each hospital established specific routes for moving COVID-19 patients. Temporary triage areas, staffed by emergency medicine staff, were created outside the hospital to separate suspected infected individuals from other emergencies (eg, trauma, stroke). Suspected COVID-19 patients presenting with mild symptoms were sent home with supportive treatment and instructed to self-isolate and tracked by phone and remotely.

Workforce and health care worker protection. A significant number of physicians and nurses were hired expeditiously from across Italy, as the needs for professionals in the region increased. Moreover, general practitioners, specialists, and other health care professionals were deployed to inpatient wards as necessary, because of the need to staff general medical and ICU wards. Palliative care physicians were charged with overseeing communications with family members and provided guidance to colleagues about managing pain and the suffering for patients in terminal states. Health care professionals received specific training on the correct donning and doffing of enhanced personal protective equipment (PPE). This training was done in class and coupled with remote live video training with an instructor at all hospital facilities. Educational activities on correct hand hygiene, waste disposal, and the use of disinfectant products were implemented within the province. During this emergency, in-person audits were done regularly by the Health Agency's Infection Control team in all hospitals and ICUs to ensure correct implementation of PPE and infection control.

\section{Community measures}

Public health surveillance strategies for COVID-19. Public health authorities defined protocols (available upon request) to classify patients according to their current symptoms and clinical comorbidities, in order to plan for their correct management and effective resource utilization (eg, inpatient admission, surveillance, home treatment, isolation).

As the number of patients arriving at the emergency department and admitted to the hospital for COVID-19 rose, an appropriate stockpiling of critical supplies was necessary, including an increased supplies of PPE and other critical devices (eg, ventilators, infusion pumps). Increased human resources (technicians, supplies) were required in laboratories to handle the dramatically increased requests for COVID-19 diagnostic tests. COVID lab test capacity rose by $136 \%$ (4860 tests per day) during the 20 days after March 24 , when only 2100 tests per day were completed. The test results of the general public and health care workers were tracked using the Pagoda dashboard.

Increase community health resources. The long-term physical, mental, and community effects of COVID-19 are not yet clear. The Emilia-Romagna region dedicated a new post-COVID care unit to extend the continuum of patient care into the community. General practitioners and the public health department had a strategic role in triaging referrals of suspected or confirmed cases, avoiding or reducing inappropriate access to emergency departments, and accelerating patient discharge from the hospital. These teams, named Care Continuity Special Units [USCA], included pediatri- cians, primary care, and public health physicians, who were put in charge of delivering care to recovering COVID-19 patients isolated at home. Each of the 7 health districts had a dedicated community management team. The mission of these activated teams was to provide care to COVID-19 patients at their residence, using in-person visits supported by newly implemented technology to assist them (eg, portable wireless ultrasound probe to diagnose pneumonia).

Isolation of suspected and confirmed cases. Isolation of patients in external facilities (eg, hotels) after hospital discharge was implemented to mitigate against further exposing other people and members of their families. Three hotels in total, one for each of the major regional health areas (North, Center, and South Modena Province), were selected and hosted 200 individuals. Home delivery of medications to COVID-19 patients in isolation was conducted by community volunteer organizations. An agreement was signed between local entrepreneurs and the Modena Healthcare Agency to host health care professionals in local hotels at no expense to the health care professionals.

\section{Discussion}

This article reports on an innovative system- and regionwide reorganization approach implemented in the Italian Modena Province to help preserve hospital and ICU capacity. These measures were deployed effectively in the region of Italy that was the second most affected by the COVID-19 pandemic during the unprecedented first wave of COVID-19 in Europe. The Modena Province leadership quickly pivoted to address urgent clinical and organizational needs and was able to prevent overwhelming local health care and ICU capacities. Unlike in other European countries, such as Germany, where the availability of ICU beds exceeded demand before the pandemic hit, ${ }^{24}$ and there was no need to triage patients during the first COVID-19 wave, ${ }^{25}$ Modena had to face an unprecedented demand for ICU beds on top of a chronic ICU bed shortage. ${ }^{7}$

Management decisions about the rapidly changing patient flow in the province were informed by the implementation of an innovative and transparent data dashboard that provided real-time feedback to hospital leaders with momentto-moment snapshots of hospitals and ICU bed utilization in all provincial health facilities. This data dashboard advised local efforts on how many COVID-19 patients could be safely handled in real time. As a result of all the rapid measures adopted in the Emilia-Romagna region the number of ICU beds more than doubled during the pandemic. When compared with the Lombardy region, the Italian region most affected by COVID-19 infected patients, the Emilia-Romagna region was able to support an effective higher rate of ICU bed availability at 2.15 beds per 10,000 inhabitants as compared with the 1.25 beds per 10,000 inhabitants in Lombardy. Fagiuoli et al report a different outcome from Bergamo's COVID experience in the adjacent Lombardy region: the tertiary hospital required converting many non-ICU wards into new ICU departments to admit sick COVID-19 patients because of the dramatic surge in very sick patients and the need for more ICU staffed beds. ${ }^{26}$ The rapid saturation of these additional units in the Lombardy region within 72 hours required extreme 
rationing of ICU care, leading to a refusal to admit sick COVID-19 patients, who were left to die in their homes because of the limited ICU resources.

The Modena Healthcare Agency's resilient leaders were able to shepherd their organizations and stakeholders through the COVID-19 crisis, with trust being critical to the recovery. The range of actions included the institution of USCA, the delivery of home medications, providing mobile units for the delivery of treatment and follow-up assessments, and maintaining an effective triage and home monitoring system for discharged patients. Importantly, implementing this community network model in a transparent and fair manner of enabled the Emilia-Romagna region to be selected by the Italian Ministry of Health as the National ICU coordinating hub for all infected COVID-19 patients. ${ }^{27}$

Modena's strong health care agency organization and resilient leadership played an essential role in the effective management of the COVID-19 crisis; they learned from early failures in optimizing staffing and equipment to support patient flow needs according to demand levels. ${ }^{7}$ For example, the idea of creating COVID-19-dedicated hospitals, as suggested by level 5 Regional guidance (Supplementary Material), was deemed impractical and, ultimately, rejected. The Modena COVID Taskforce quickly realized that all the hospitals in the province had an emergency care unit that represented the only community access point for acute care. Moreover, at the beginning of the epidemic, hospitals were reluctant to receive COVID-19-positive citizens as these patients put additional organizational pressure on units that were already struggling to accommodate a higher than expected volume of sick patients.

\section{Lessons learned}

As the ninth-month mark after the first confirmed COVID-19 pandemic case in Italy nears, 5 lessons can be learned from the Modena experience for other jurisdictions to consider:

1) Accelerating the pace of innovation and new technology, despite being in the midst of a pandemic. Community medical services-including diagnosis, treatment, and isolation delivered at the homes of patients-were crucial to effectively reducing the pressure on and access to hospitals, and minimize the spread of the virus. The Modena web-based triage tool connected people with the appropriate level of care, whether speaking with an emergency department clinician, or staying home. These tools were deployed rapidly, providing patients the help they needed in the early days of the pandemic.

2) Strong community-hospital partnership. Immediate hospital admission of all suspected and confirmed cases was not sustainable in the long run. An effective alternative option was the use of hotels and community resources that were turned into patient isolation hubs. This contributed to strengthening public confidence and building social trust in Modena's leaders.

3) Better data support and decision-making. The automated centralized dashboard for the monitoring of patient flow was essential to enable real-time, optimal decisionmaking. The use of the Pagoda data dashboard has since become routine practice for all COVID and non-COVID care. Just-in-time performance indicators and predictive intelligence played an essential role in guiding rational and need-based decision-making. Key stakeholders agreed on indicators to measure performance, ${ }^{28}$ in a transparent and timely manner. The data was monitored and reported on in order to transparently strategize actions toward a pandemicresilient health system. ${ }^{29}$

4) You can never be overprepared. Having organizational preparedness plans in place is essential to respond effectively to emergencies so that effective containment measures can be implemented effectively. Strategic protocols were developed to allow elective care activities to restart, with detailed procedures for a rapid return to only emergency activities as needed. ${ }^{30}$ In preparation for the second wave of the COVID pandemic, the Modena Healthcare Agency has hired and trained more health professionals, increased the number of beds permitted in each ICU unit, installed ad hoc technologies, enforced community USCA, introduced the use of a thermoscanner at hospital entrances, and refined the diagnostic performance of COVID-19 molecular tests.

5) The safety of the Modena health care workforce remains the number one priority. Nine months into the pandemic, health care workers are still becoming infected and dying despite wearing PPE, raising indignation about inadequate PPE, poor understanding of the dangers of reusing PPE, and a need for a deeper understanding of practical infection control practices in hospitals and clinics. ${ }^{31}$ A robust health care worker protection and risk stratification plan is needed; ongoing self- and system monitoring of health care worker health conditions is essential. ${ }^{26}$

\section{Limitations}

There are limitations to this study. The study's findings concern only a single Italian province and region, and may limit the generalizability of the results. The region's ICU bed utilization continues to be held up as a success nationally and internationally and is being audited rigorously, given the ongoing pandemic. A more extensive epidemiological investigation is needed to provide a robust analysis of the effectiveness of the range of steps we have described that helped preserve the health systems resiliency.

The research team recognize that it is not possible to disentangle the contribution of the hundreds of actions that were implemented to obtain effective population health management outcomes in Modena. Moreover, these actions were implemented under a framework of a local and national lockdown and have been praised for their effectiveness by the World Health Organization. They contributed significantly to limiting the diffusion of COVID-19 into the Modena community. ${ }^{32,33}$ Nevertheless, as the second wave of COVID is hitting Italy, critical differences have emerged in regard to ICU bed management and, therefore, occupancy in the different Italian regions. ${ }^{34,35}$

\section{Conclusions}

This article reports on the actions undertaken inside the hospital and in close concert with the actions taken at the meso and macro levels of the Modena Province in successfully addressing the first wave of the COVID-19 pandemic. The actions included strengthening the network of resources available in the community-from administering chemotherapy to cancer patients right in their own home, to providing 
residents with coronavirus testing and educational resources at home-which greatly helped to reduce the burden of care on hospitals while protecting health care workers from being harmed. ${ }^{36-38}$

The automated, centralized data dashboard for monitoring patient flow enabled the correct real-time management of patient admissions and ICU bed optimization in the Modena Province. These findings support the importance of strong leadership willing to use and implement a centralized data dashboard, instrumental in managing bed utilization and mitigating a larger public health crisis. The region was able to avoid overwhelming its provincial hospitals, helping to minimize the inevitable pitfalls related to a surge in ICU bed demand, which led to higher mortality in other adjacent, similarly equipped regions. ${ }^{39}$

The measures adopted by the Modena Province contributed to the containment of the pandemic in Italy and most likely prevented major outbreaks in other regions. Some of the measures undertaken by the Modena Province have been implemented in other European and non-European countries. However, all of these measures were quickly and rapidly implemented (in a short time frame between March and April 2020) not as a single practice, but together in a concerted manner. The effective leadership exhibited by the Modena Province is supported by the effectiveness of the seamless and collective effort in managing the first severe outbreaks of the virus.

Modena's health care managers were able to reach the goal rapidly: reducing the Rho value with respect to the predictions, avoiding ICU overload and rationing of medical care. As a result, these rapid decisions and actions made the Modena region of Emilia-Romagna, the model region in Italy, and justified their selection by the Italian Ministry of Health as the National ICU coordinating hub for all sick COVID-19 patients in Italy.

The results of this study offer potential insights to many countries, that are continuing to see a rapid, dramatic rise in the number of hospitalized COVID-19 patients, threatening to overwhelm ICU units. The findings from this study can inform decision makers in thier responses to quickly mobilize and institute specific approaches for case identification, containment, contact tracing, and smart resource allocation. We believe this approach can help protect the vitality of their health systems, and importantly engender trust in their systems and leaders.

\section{Authors' Contributions}

Drs. Romani, Ferrara, and Lucà: study concept and design. Drs. Romani, Cobianchi, Modenese, Massaro, and Lucà: data collection and analysis. Drs. Ferrara, Dal Mas, Massaro, Cobianchi, Barcellini, Ricciardi, Barach, and Lucà: critical revision of the manuscript for important intellectual content. All authors approved the final version of the manuscript. Dr. Paul Barach, Dr. Rossella Lucà, and Dr. Maria Ferrara substantially contributed as co-last authors.

\section{Author Disclosure Statement}

The authors declare that there are no conflicts of interest.

\section{Funding Information}

No funding was received for this article.

\section{Supplementary Material}

Supplementary Data

Supplementary Figure S1

Supplementary Figure S2

Supplementary Figure S3

Supplementary Figure S4

Supplementary Table S1

\section{References}

1. WHO-China Joint Mission on Coronavirus Disease 2019 (COVID-19) report. WHO. 2020. https://www.who.int/ docs/default-source/coronaviruse/who-china-joint-missionon-covid-19-final-report.pdf Accessed April 13, 2020.

2. Coronavirus COVID-19 Global Cases. 2020. https:// coronavirus.jhu.edu/map.html Accessed March 30, 2020

3. Livingston E, Bucher K, Rekito A. Coronavirus disease 2019 and influenza 2019-2020. JAMA 2020;323: 1122.

4. Meissner P, Wulf T. The development of strategy scenarios based on prospective hindsight: an approach to strategic decision making. J Strategy Manage 2015;8:176-190.

5. Cobianchi L, Dal Mas F, Peloso A, et al. Planning the full recovery phase: an antifragile perspective on surgery after COVID-19. Ann Surg 2020;272:e296-e299, doi: 10.1097/ SLA.0000000000004489

6. Golinelli D, Bucci A, Toscano F, Filicori F, Fantini MP. Real and predicted mortality under health spending constraints in Italy: a time trend analysis through artificial neural networks. BMC Health Serv Res 2018;18:671.

7. Grasselli G, Pesenti A, Cecconi M. Critical care utilization for the COVID-19 outbreak in Lombardy, Italy: Early experience and forecast during an emergency response. JAMA 2020;323:1545-1546.

8. Liao X, Wang B, Kang Y. Novel coronavirus infection during the 2019-2020 epidemic: preparing intensive care units-the experience in Sichuan Province, China. Intensive Care Med 2020;46:357-360.

9. Sorveglianza integrata COVID-19: i principali dati nazionali. 2020. https://www.epicentro.iss.it/coronavirus/sars-cov-2sorveglianza-dati Accessed March 26, 2020.

10. Istituto Nazionale di Statistica. www.istat.it/ Accessed April 13, 2020.

11. Ministero della Salute, Open data. 2020. www.dati.salute .gov.it/dati/homeDataset.jsp Accessed April 13, 2020.

12. Dipartimento della Protezione Civile. Accessibilità e catalogo dei dati, metadati e banche dati. Department of Italian Civil Protection. Accessibility and catalogue of data, metadata and databases. http://www.protezionecivile.gov.it/amministrazionetrasparente/altri-contenuti/accessibilita-e-catalogo-dei-datimetadati-e-banche-dati Accessed April 13, 2020.

13. Coronavirus. Posti letto, piano della Regione: già allestiti 1.300 posti, pronti ad arrivare a 3.120. 2020. https://www .regione.emilia-romagna.it/notizie/2020/marzo/coronavirusposti-letto-piano-della-regione-gia-allestiti-1300-posti-prontiad-arrivare-a-3120 Accessed December 8, 2020.

14. 118-emergency transportation. 2020. https://www.118er .it/emiliaest/ Accessed December 8, 2020.

15. Li MY, Graef JR, Wang L, Karsai J. Global dynamics of a SEIR model with varying total population size. Math Biosci 1999;160:191-213.

16. Li MY, Muldowney JS. Global stability for the SEIR model in epidemiology. Math Biosci 1995:155-164. doi: 10.1016/0025-5564(95)92756-5. PMID: 7881192. 
17. Peng L, Yang W, Zhang D, Zhuge C, Hong L. Epidemic analysis of COVID-19 in China by dynamical modeling. arxiv 2020;2002.06563.

18. Sprung CL, Zimmerman JL, Christian MD, et al. Recommendations for intensive care unit and hospital preparations for an influenza epidemic or mass disaster: summary report of the European Society of Intensive Care Medicine's Task Force for intensive care unit triage during an influenza epidemic or mass disaster. Intensive Care Med 2010;36: $428-443$.

19. Lurie N, Carr BG. The role of telehealth in the medical response to disasters. JAMA Intern Med 2018;178:745746.

20. de Girolamo G, Cerveri G, Clerici M, et al. Mental health in the coronavirus disease 2019 emergency-the Italian response. JAMA Psychiatry 2020;77:974-976.

21. Starace F, Ferrara M. COVID-19 disease emergency operational instructions for Mental Health Departments issued by the Italian Society of Epidemiological Psychiatry. Epidemiol Psychiatr Sci 2020;29:e116.

22. CoVID-19 in gravidanza, parto e puerperio. 2020. www .saperidoc.it/flex/cm/pages/ServeBLOB.php/L/IT/IDPagina/ 1382 Accessed March 30, 2020.

23. The treatment of cancer patients during COVID19 pandemia. 2020. https://www.aiom.it/wp-content/uploads/2020/ 03/20200325_COVID19_SICO-AIOM-AIRO.pdf Accessed April 21, 2020.

24. Chazan G. Oversupply of hospital beds helps Germany to fight virus. Financial Times 2020.

25. Stang A, Stang M, Jockel KH. Estimated use of intensive care beds due to COVID-19 in Germany over time. Dtsch Arztebl Int 2020;117:329-335.

26. Fagiuoli S, Lorini FL, Remuzzi G. Covid-19 Bergamo Hospital Crisis U. Adaptations and Lessons in the Province of Bergamo. N Engl J Med 2020;382:e71.

27. News online. Coronavirus, Region Emilia-Romagna becomes the national Hub for Intensive Care. https://salute.regione .emilia-romagna.it/notizie/regione/2020/aprile/coronaviruslemilia-romagna-diventa-hub-nazionale-per-la-terapiaintensiva Accessed December 8, 2020.

28. Ferrara M, Mathis WS, Cahill DJ, Pollard J, Srihari HV. Early detection of the schizophrenia(s): a population health approach. In: Hardy KV, Ballon JS, Noordsy DL, Adelsheim S, American Psychiatric Association, eds. Intervening early in psychosis: a team approach. First edition. Washington, DC: American Psychiatric Association Publishing; 2019:xxi, 437 pages.

29. Kringos D, Carinci F, Barbazza E, et al. Managing COVID19 within and across health systems: why we need per- formance intelligence to coordinate a global response. Health Res Policy Syst 2020;18:80.

30. Zanardo M, Schiaffino S, Sardanelli F. Bringing radiology to patient's home using mobile equipment: a weapon to fight COVID-19 pandemic. Clin Imaging 2020;68:99-101.

31. Van Zundert T, Barach P, Van Zundert AAJ. Revisiting safe airway management and patient care by anaesthetists during the COVID-19 pandemic. Br J Anaesth 2020;125: P863-P867.

32. Lavezzo E, Franchin E, Ciavarella C, et al. Suppression of a SARS-CoV-2 outbreak in the Italian municipality of Vo'. Nature 2020;584:425-429.

33. Vicentini C, Bordino V, Gardois P, Zotti CM. Early assessment of the impact of mitigation measures on the COVID-19 outbreak in Italy. Public Health 2020;185:99-101.

34. Newspaper online. Covid bed terminated in Intensive care Units in Lombardy: the Fiera Hospital in Milan opens back its doors. Retrieved from https://www.milanotoday.it/ attualita/coronavirus/ospedale-fiera-23-ottobre-2020.html Accessed December 8, 2020.

35. Covid-19-Situazione in Italia. 2020. www.salute.gov.it/ portale/nuovocoronavirus/dettaglioContenutiNuovoCorona virus.jsp?lingua $=$ italiano $\& \mathrm{id}=5351 \&$ are $a=$ nuovoCorona virus\&menu=vuoto Accessed October 23, 2020.

36. Pisano GP, Sadun R, Zanini M. Lessons from Italy's response to coronavirus. Harvard Business Review 2020.

37. Orians C, Rose S, Hubbard B, et al. Strengthening the capacity of local health agencies through community-based assessment and planning. Public Health Rep 2009;124:875882.

38. Bouri N, Sell TK, Franco C, Adalja AA, Henderson DA, Hynes NA. Return of epidemic dengue in the United States: implications for the public health practitioner. Public Health Rep 2012;127:259-266.

39. Rhodes A, Ferdinande P, Flaatten H, Guidet B, Metnitz PG, Moreno RP. The variability of critical care bed numbers in Europe. Intensive Care Med 2012;38:1647-1653.

Address correspondence to:

Maria Ferrara, MD

Department of Psychiatry

Yale University, School of Medicine 34 Park Street New Haven, CT 06519

USA

E-mail: maria.ferrara@yale.edu 